\title{
Phase Transitions: A Challenge for Intertheoretic Reduction?
}

\author{
Patricia Palacios * \\ Department of Philosophy \\ University of Salzburg
}

\begin{abstract}
In this paper, I analyze the extent to which classical phase transitions, both first-order and continuous, pose a challenge for intertheoretic reduction. My main contention is that phase transitions are compatible with reduction, at least with a notion of inter-theoretic reduction that combines Nagelian reduction and what Nickles (1973) called reduction ${ }_{2}$. I also argue that, even if the same approach to reduction applies to both types of phase transitions, there is a crucial difference in their physical treatment. In fact, in addition to the thermodynamic limit, in the case of continuous phase transitions there is a second infinite limit involved that is related with the number of iterations in the renormalization group transformation. I contend that the existence of this second limit, which has been largely underappreciated in the philosophical debate, marks an important difference in the reduction of first-order and continuous phase transitions and also in the justification of the idealizations involved in these two cases.
\end{abstract}

\section{Introduction}

Phase transitions are sudden changes in the phenomenological properties of a system. Some common examples include the transition from liquid to gas, from a normal conductor to a superconductor, or from a paramagnet to a ferromagnet. Nowadays phase transitions are considered one of the most interesting and controversial cases in the analysis of inter-theory relations. This is because they make particularly clear the constitutive role played by idealizations in inferring macroscopic behavior from a theory that describes microscopic interactions. In fact, it appears that statistical mechanics a well-established microscopic theory - cannot account for the behavior of phase transitions as described by thermodynamics - a macroscopic theory without the help of infinite idealizations in the form of mathematical limits.

*email: patricia.palacios@sbg.ac.at 
In the discussion on phase transitions, physicists and philosophers alike have mainly been concerned with the use of the thermodynamic limit, an idealization that consists in letting the number of particles as well as the volume of the system go to infinity. For many authors (e.g. Batterman 2005; Bangu 2009, Bangu 2011; Batterman 2011, Morrison 2012, Batterman 2017) this idealization has an important philosophical consequence: it implies that phase transitions are emergent phenomena. As a result, they claim that such phenomena present a challenge for the reduction of thermodynamics to statistical mechanics. In particular, the case of continuous phase transitions that implements renormalization group (RG) techniques is regarded as especially problematic for the reduction of phase transitions (e.g. Batterman 2011, Morrison 2012, Batterman 2017).

On the other hand, numerous other authors (e.g. Butterfield 2011; Butterfield and Bouatta 2011; Norton 2012; Callender 2001; Menon and Callender 2013) have rejected this conclusion, arguing that the appeal to the infinite limit does not represent a problem for inter-theory reduction. Most of them (Butterfield 2011, Butterfield and Bouatta 2011, Norton 2012) have even argued that phase transitions, including continuous phase transitions, are paradigmatic examples of Nagelian reduction, whereby reduction is understood in terms of logical deduction.

In this paper I take the side of the reductionists, but contra the "received" reductionist position, I claim that the Nagelian model is not sufficient to describe the relation between the thermodynamics of phase transitions and statistical mechanics. Instead, I argue that a combination of Nagelian reduction and what Nickles (1973) called reduction 2 provides us with a much more natural framework for both first-order and continuous phase transitions. I will also argue that, even if the same approach to reduction applies to both kinds of transitions, there is a crucial difference between them: in the case of continuous phase transitions there is a second infinite limit involved that is related with the number of iterations in the renormalization group transformation. I will contend that the existence of this second infinite limit, which has been largely underappreciated in the philosophical discussion, marks an important difference in the reduction of first-order and continuous phase transitions and also in the justification of the idealizations involved in these two cases, for example objections related with the singular nature of the thermodynamic limit that apply to the case of first-order phase transitions do not apply to the case of continuous phase transitions.

To reach my goal, I organize this paper as follows. In the next section (Section 2), I describe the physics of phase transitions, outlining how statistical mechanics recovers thermodynamical behavior. Here I emphasize that in the RG treatment of continuous phase transitions, apart from the thermodynamic limit, there is a second infinite limit involved. Subsequently (Section 3.1-3.2), I further develop the concept of inter-theory reduction, distinguish- 
ing between different kinds of reduction and stating the formal and empirical criteria for each of them. I argue then (Section 3.3) that the most suitable model for the reduction of both first-order and continuous phase transitions is a combination of Nagelian reduction and what Nickles (1973) called reduction 2 , more specifically limiting reduction. Based on that notion of reduction, I contend (Section 4.1) that, despite some objections regarding the "singular nature" of the thermodynamic limit (Batterman 2005, 2009), first-order phase transitions satisfy the criteria for inter-theory reduction. Later (Section 4.2), I argue that the objections related with the singular nature of the thermodynamic limit do not apply to the case of continuous phase transitions because the set of limiting operations is not singular. However, I will point out that there is a different problem in the continuous phase transitions related with the application of the second infinite limit in finite systems. In Section 4.3, I will offer a solution to this problem based on topological and numerical arguments.

\section{From Statistical Mechanics to the Thermody- namics of Phase Transitions}

Statistical mechanics aims to account for the macroscopic behavior typically described by thermodynamics in terms of the laws that govern microscopic interactions. In the philosophical literature, the reproduction of the thermodynamic results by statistical mechanics is generally referred to in terms of reduction. In this section, I will describe how statistical mechanics recovers the thermodynamic behavior of phase transitions and will explain why phase transitions are an interesting and puzzling case for the project of reducing thermodynamics to statistical mechanics.

\subsection{The Thermodynamics of Phase Transitions}

In thermodynamics, phases correspond to regions of the parameter space (known as a phase diagram) where the values of the parameters uniquely specify equilibrium states. Phase boundaries, in contrast, correspond to values of parameters at which two different equilibrium states can coexist. The coexistence of states expresses itself as discontinuities of thermodynamic quantities, like volume, which are related to the first derivatives of the free energy with respect to a parameter such as pressure or temperature. If the system goes from one phase to another intersecting a phase boundary, the system is said to undergo a first-order phase transition. This name is due to the fact that the discontinuous jumps occur in the first derivatives of the free energy. On the other hand, if the system moves from one phase to another without intersecting any coexistence line, the system is said to undergo a continuous phase transition, in which case there are no discon- 
tinuities involved in the first derivatives of the free energy but there are divergences in the response functions (e.g. specific heat, susceptibility for a magnet, compressibility for a fluid). An example of a first-order phase transition is the passage from liquid water to vapor at the boiling point, where the quantities that experience discontinuous jumps are entropy and volume, which are first derivatives of the free energy with respect to temperature and pressure respectively. An example of continuous phase transition instead is the transition in magnetic materials from the phase with spontaneous magnetization - the ferromagnetic phase - to the phase where the spontaneous magnetization vanishes - the paramagnetic phase (Figure 1).

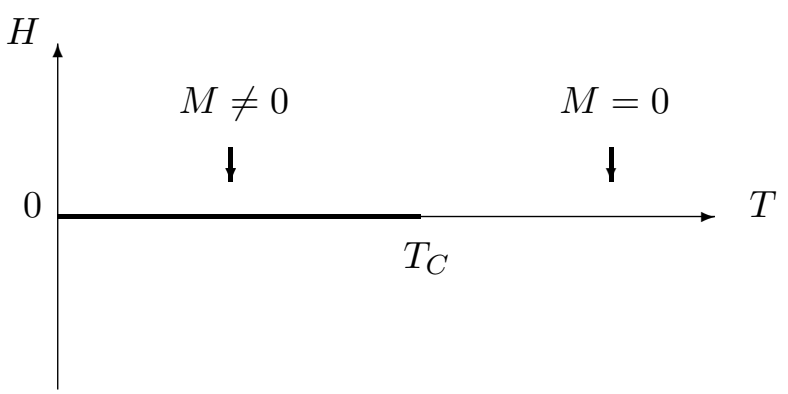

Figure 1: Phase diagram for the paramagnetic-ferromagnetic transition. Here $H$ is the external magnetic field and $T$ the temperature. At the transition or critical point $T_{C}$ the spontaneous magnetization $M$ vanishes.

Although both first-order and continuous phase transitions are of great interest for the project of reducing thermodynamics to statistical mechanics, the latter kind is considered to be more troubling than the former. The reason is that continuous phase transitions have characteristic properties that are much more difficult to recover from statistical mechanics than firstorder phase transitions. One of those properties is that, in the vicinity of a continuous phase transition, measurable quantities depend upon one another in a power-law fashion. For example, in the ferromagnetic-paramagnetic transition, the net magnetization $M$, the magnetic susceptibility $\chi$, and the specific heat $C$ depend on the reduced temperature $t=\left(T-T_{c}\right) / T_{c}$ (the temperature of the system with respect to the critical temperature $T_{c}$ ) as follows:

$$
M \sim|t|^{\beta}, C \sim|t|^{-\alpha}, \chi \sim|t|^{-\gamma},
$$

where $\beta, \alpha, \gamma$ are the critical exponents. Another remarkable property of continuous phase transitions is that radically different systems, such as fluids 
and ferromagnets, have exactly the same values for the critical exponents, a property known as universality.

Finally, continuous phase transitions are also characterized by the divergence of some physical quantities at the transition or critical point. The critical exponents $\alpha$ and $\gamma$ are typically (although not always) positive, so that the power laws that have negative exponents (and the corresponding quantities like specific heat and susceptibility) diverge as $T \rightarrow T_{c}$. The divergence of the magnetic susceptibility $\chi$ implies the divergence of the correlation length $\xi$, a quantity that measures the distance over which the spins are correlated, which also obeys power-law behavior: $\xi \sim|t|^{-\nu}$. The divergence of the correlation length is perhaps the most important feature of continuous phase transitions because it involves the loss of a characteristic scale at the transition point and thus provides a basis for universal behavior.

The calculation of the experimental values of critical exponents - or adequate relations among them - together with a good account of universality has been one the major challenges of statistical mechanics. We will see in Section 2.3 that in order to provide such an account, it was necessary to appeal to infinite idealizations and to RG methods, an entirely new theoretical framework, which basically consists in reducing the number of effective degrees of freedom of the system.

\subsection{The Importance of the Thermodynamic Limit in the De- scription of First-Order Phase Transitions}

We saw in the previous section that the macroscopic behavior of first-order phase transitions is defined in terms of singularities or non-analyticities in the first derivatives of the free energy. Gibbsian statistical mechanics offers a precise definition of the free energy $F$, given by:

$$
F\left(K_{n}\right)=-\kappa_{B} T \ln Z,
$$

where $K_{n}$ is the set of coupling constants, $\kappa_{B}$ is the Boltzmannian constant, $T$ is the temperature, and $Z$ is the canonical partition function, defined as the sum over all possible configurations:

$$
Z=\sum_{i} e^{\beta H_{i}} .
$$

When trying to use statistical mechanics to recover the non-analyticities that describe phase transitions in thermodynamics, the following problem arises. Since the Hamiltonian $H$ is usually a non-singular function of the degrees of freedom, it follows that the partition function, which depends on the Hamiltonian, is a sum of analytic functions. This means that neither the free energy, defined as the logarithm of the partition function, nor its derivatives can have the singularities that characterize first-order phase transitions in thermodynamics. Taking the thermodynamic limit, which consists 
of letting the number of particles as well as the volume of the system go to infinity $N \rightarrow \infty, V \rightarrow \infty$ in such a way that the density remains finite, allows one to recover those singularities. In this sense, the use of this limit appears essential for the recovery of the exact values of the thermodynamic quantities.

\subsection{The Appeal to a Second Infinite Limit in the Case of Continuous Phase Transitions}

In an ideal scenario, one would expect to perform a direct calculation of the partition function. Unfortunately, analytic calculations of the partition functions have been performed only in particular models with dimension $D=1$ or $D=2$; for all other cases, one requires approximation techniques. ${ }^{1}$ The most useful approximation for the case of first-order phase transitions is the mean field approximation, which employs the assumption that each spin acts as if it were independent of the others, feeling only the average mean field. Although the mean field approximation proved to be successful in some cases of first order phase transitions, experiments have shown that this account fails to give accurate predictions for the case of continuous phase transitions, in which the correlation length diverges. It is believed that this failure is due to the fact that mean field theories neglect fluctuations whereas fluctuations govern the behavior near the critical point (Goldenfeld 1992).

A more complete account of continuous phase transitions requires the use of RG methods. These methods are mathematical and conceptual tools that allow one to solve a problem involving long-range correlations by generating a succession of simpler (generally local) models. The goal of these methods is to find a transformation that successively coarse-grains the effective degrees of freedom but keeps the partition function and the free energy (approximately) invariant. The usefulness of RG methods lies in the fact that one can compute the critical exponents and other universal properties without having to calculate the free energy. These methods also allow one to account for universality, the remarkable fact that entirely different systems behave qualitatively and quantitatively in the same way near the critical point.

To give a specific illustration of RG methods, let us consider a block spin transformation for a simple Ising model on the two-dimensional square lattice with distance $a$ between spins. ${ }^{2}$ Here, the spins have two possible values, namely \pm 1 . If it is assumed that the spins interact only with an

\footnotetext{
${ }^{1}$ The first and most famous exact solution of the partition function is the Onsager solution for an Ising model of dimension $\mathrm{D}=2$.

${ }^{2}$ For simplicity, I am going to restrict here the analysis to real-space renormalization. However, the same conclusions apply to momentum-space renormalization. For details on the difference between real-space and momentum space-renormalization, see Wilson and Kogut (1974) and Fisher (1998). For a philosophical account on the difference between those two frameworks see Franklin (2017)
} 
external magnetic field $h$ and with their nearest neighbors through the exchange interaction $K$ (meaning that the coupling constants are only $K$ and $h$ ), the Hamiltonian $H$ for the model is given by:

$$
H=-K \sum_{i j}^{N} S_{i} S_{j}+-h \sum_{i} S_{i} .
$$

By applying the majority rule, which imposes the selection of one state of spin based on the states of the majority of spins within a block, one can replace the spins within a block of side la by a single block spin. Thus, one obtains a system that provides a coarse-grained description of the original system.

If one assumes further that the possible values for each block spin $S_{I}$ are the same as in the Ising model, namely \pm 1 , and also that the block spins interact only with nearest neighbor block spins and an external field, the effective Hamiltonian $H^{\prime}$ will have the same form as the original Hamiltonian $H$ :

$$
H^{\prime}=-K^{\prime} \sum_{I J}^{N l^{-d}} S_{I} S_{J}+-h^{\prime} \sum_{I} S_{I} .
$$

Formally, this is equivalent to applying a transformation $\mathrm{R}$ to the original system, so that $H^{\prime}=R[H]$, in which the partition function and the free energy remain approximately invariant. ${ }^{3}$

Although the systems described by $H$ and $H^{\prime}$ have the same form, the correlation length in the coarse-grained system $\xi\left[K^{\prime}\right]$ is smaller than the correlation length $\xi[K]$ of the original system. This follows from the fact that the correlation length in the effective model is measured in units of the spacing $l a$ whereas the correlation length in the original system is measured in units of the spacing $a$. In other words, the correlation length is rescaled by a factor $l$. The expression that relates the correlation lengths of the two systems is:

$$
\frac{\xi[K]}{l}=\xi\left[K^{\prime}\right] .
$$

After $n$ iterations of the RG transformation, the characteristic linear dimension of the system is $l^{n}$. Thus the correlation lengths in the sequence of coarse-grained models vary according to:

$$
\xi[K]=l \xi\left[K^{\prime}\right]=\ldots=l^{n} \xi\left[K^{(n)}\right] .
$$

\footnotetext{
${ }^{3}$ The previous example captures the spirit of real-space RG methods. However in practice RG transformations consist of complicated non-linear transformations that do not preserve the form of the original Hamiltonian. This allows for the possibility that new local operators are generated during the RG transformation (Details in Goldenfeld 1992, p. 235).
} 
The idea is that one iterates the RG transformation until fluctuations at all scales up to the physical correlation length $\xi$ are averaged out. In many cases, this involves numerous iterations. Details elsewhere, e.g. Le Bellac, Mortessagne, and Batrouni 2006, Sec. 4.4.3; Goldenfeld 1992, Sec. 9.3.

It follows from equation (6) that for a large correlation length, the number of iterations should be large. If the original correlation length $\xi[K]$ is infinite and we want to eliminate all effective degrees of freedom, i.e. we want the effective correlation length to be small, then we are forced to take the limit $n \rightarrow \infty$ in the right hand side of equation (6) such that the following expression holds:

$$
\xi[K]=\lim _{n \rightarrow \infty} l^{n} \xi\left[K^{(n)}\right]=\infty
$$

This result is important because it demonstrates the existence of two different infinite limits involved in the traditional approach to phase transitions. The first is the thermodynamic limit that takes us to a system with an infinite correlation length. The second is the limit for the number of RG iterations going to infinity that takes us to a fixed point Hamiltonian, i.e. the Hamiltonian with the coupling constants equal to their fixed point values: $\left[K^{*}\right]=R\left[K^{*}\right]$. These fixed points can be also thought of as stationary or limiting distributions to which the renormalization group trajectories converge after infinite iterations of the RG transformation $n \rightarrow \infty$. It is important to emphasize here that this does not mean that the two limits are "essential" to find non-trivial fixed points, rather it means that if we take the thermodynamic limit, then we need to take a second infinite limit in which the number of RG iterations go to infinity. This is related with the non-commutability of the two limits that I will explain in more detail in Section 4.3.

Although the iteration of the RG transformation preserves the symmetries of the original system, it does not preserve the value of the original Hamiltonian, and, therefore, it does not preserve the value of the set of coupling constants $[K]$ associated with the corresponding Hamiltonians. Thus, the iteration of the RG transformation can be thought of as describing a sequence of points moving in a space of coupling constants $K^{n}$ or a corresponding space of Hamiltonians $H$. If the sequence describes a system at the critical point, after infinite iterations $n \rightarrow \infty$ it will converge to a non-trivial fixed point $\left[\mathrm{K}^{*}\right]$ given by:

$$
\left[K^{*}\right]=R\left[K^{*}\right]
$$

The other possible fixed points are trivial, namely $K=0$ and $K=\infty$, which correspond to low and high temperature fixed points respectively.

At fixed points the coupling constants remain invariant under the transformation. Therefore, varying the length scale does not change the value of the Hamiltonian and therefore brings us to a physically identical system. 
This latter feature associates fixed points with the property of scale invariance, which means that the system looks statistically (and physically) the same at different scales.

It has been shown that by linearizing in the vicinity of the fixed point, one can calculate the values of the critical exponents and the relations between them (Details in Goldenfeld 1992, Sec. 9; Domb 2000, Sec. 7; Sornette 2000, Sec. 11). This is remarkable because it demonstrates that the critical exponents are solely controlled by the RG trajectory near the fixed point and that one does not need to calculate the free energy to determine the behavior of the system in the vicinity of the critical point. This means also that the initial values of the coupling constants do not determine the critical behavior. The latter constitutes the origin of the explanation of universality because it tells us that systems that flow towards the same fixed point are governed by the same critical exponents, even if they are originally described by different coupling constants. The systems that flow towards the same fixed point - that are in the basin of attraction of the fixed point - are said to be in the same universality class.

In summary, we have seen that the recovery of the thermodynamic properties from statistical mechanics usually involves: i) first, the introduction of particular assumptions (e.g. lattice structure, a particular kind of degrees of freedom, ranges of values of the degrees of freedom, and dimension) that allow one to build a specific model (Ising model in our case study); ii) second, the assumption of the thermodynamic limit, which brings us to a fine-grained system with infinite number of particles and infinite correlation length; ${ }^{4}$ and iii) finally, the assumption of a second infinite limit that consists of an infinite number of iterations of a coarse-graining transformation. This limit takes us to a fixed point Hamiltonian that represents a coarsegrained model. After those steps are made, the most important statistical mechanical approaches make accurate predictions of the behavior of continuous phase transitions and explain universal behavior. Figure 2 illustrates this process. Notice, however, that in the case of first-order phase transitions, one could in principle derive the thermodynamic behavior just after taking the first limit. ${ }^{5}$

\footnotetext{
${ }^{4}$ Recently, Norton (2012) has challenged the appeal to an infinite system in the theory of phase transitions. His contention is that the limit system would have properties that are not suitable to describe phase transitions, such as the violation of determinism and energy conservation. This point is relevant for his distinction between idealizations and approximations, which led him to the conclusion that phase transitions are a case of approximation and not idealization. Since we are trying to make a different point here, we are going to adhere to the standard façon de parler that refers to the existence of an "infinite system" (e.g. Kadanoff 2009; Fisher 1998; Butterfield 2011). This does not mean that our view is incompatible with Norton's view.

${ }^{5}$ One should bear in mind that although RG methods are not required to infer the behavior of first-order phase transitions, they can be (and have been) used to describe these kinds of transitions as well. See Goldenfeld (1992, Sec. 9).
} 


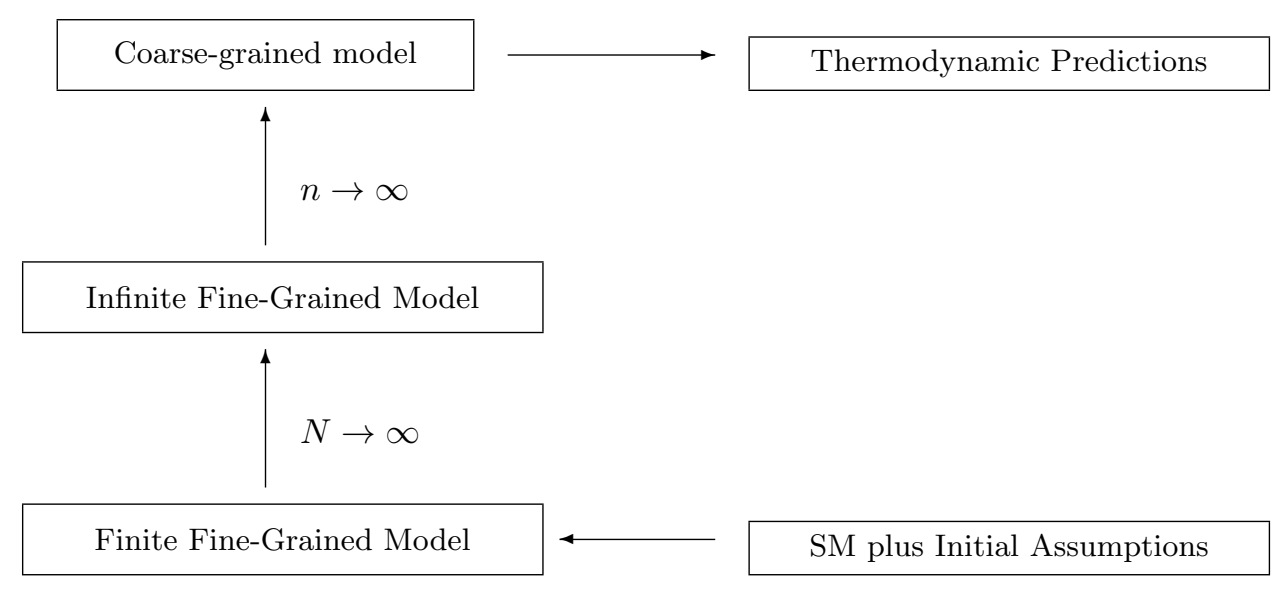

Figure 2: Inter-theory relation for traditional approaches to continuous phase transitions.

\section{The Concept of Intertheoretic Reduction}

What has been at stake in the philosophical debate around phase transitions is whether the thermodynamic description of these phenomena reduces to statistical mechanics. Even if the previous section showed that statistical mechanics can reproduce the non-analyticities that describe phase transitions in thermodynamics, the appeal to infinite limits throws suspicion on the legitimacy of such a reduction. The main aim of the following sections is to evaluate whether the infinite idealizations mentioned in Section 2 are compatible with the reduction of phase transitions. However, given that the term "reduction" is notoriously ambiguous, before we can assess this issue, some clarifications as to how this term is explicated in this context are necessary. This is the task of the present section.

\subsection{On the Goals of Intertheoretic Reduction}

Since we are interested in relating the thermodynamic treatment of phase transitions with another theory - statistical mechanics - that aims to describe the same phenomena, we are treating phase transitions as a potential case of inter-theory reduction, where reduction is taken as a relation between two theories (or parts of theories) ${ }^{6}$ Very often, especially in the discussion around phase transitions, inter-theory reduction is equated with the

\footnotetext{
${ }^{6}$ This kind of reduction is to be distinguished from other types of reduction such as whole-parts reduction. (See Norton (2012) for a clear distinction between these two kinds of reduction).
} 
Nagelian model of reduction (or revised versions of it), which understands reduction in terms of logical deduction. For reasons that will become clear below, here I consider a more general notion of inter-theory reduction that is not restricted to the Nagelian model. A definition that will be useful in the present context is the one suggested by Rosaler (2017), who understands reduction as

the relationship whereby one description successfully models all real behaviors that are well-modeled on another - or, somewhat more concisely, the relationship whereby one description subsumes the domain of applicability of another. (2)

According to this view, the reduction of a theory $T_{h}$ (generally taken to be the high level theory) to another $T_{l}$ (generally taken to be the low level theory) implies that all real behaviors that can be accurately modeled by $T_{h}$ can be modeled at least as accurately by $T_{l}$. A virtue of this definition is that it is general enough to account for different models of intertheory reduction that have been present in the philosophical literature (the Nagelian model being one of them). These different models of reduction will be regarded as different strategies to reach the main goal of intertheory reduction which is the subsumption of the domain of applicability of a theory by another. Another virtue of this definition is that it is compatible with the variety of scientific and philosophical functions that have been attributed to reduction. In fact, domain subsumption can amount to ontological economy, i.e. the thesis that the behavior described by $T_{h}$ is nothing over and above the behavior described by $T_{l}$, to the explanation of one theory by another or to heuristic and justificatory functions, depending on the case.

In the case of phase transitions, one would like to demonstrate that statistical mechanics can account for the behavior that is successfully described by thermodynamics first for the purpose of justifying the success of thermodynamics from the point of view of statistical mechanics (a theory that we believe is more correct), and second to support the ontological thesis that phase transitions are nothing over and above the result of the behavior of atomic interactions. Having said that, the question is now which approach to reduction, if any, will allow us to show that statistical mechanics reduces the behavior of thermodynamic phase transitions in the previous sense. In the philosophical discussion, most philosophers defending the reduction of phase transitions have argued that phase transitions are a case of Nagelian reduction (Butterfield 2011, Butterfield \& Bouatta 2011, Norton 2012). In contrast to these authors, I will argue later that the Nagelian model does not suffice to provide an adequate model for the case of phase transitions and that a combination of Nagelian reduction and what Nickles called reduction 2 provides us with a much more natural framework for the reduction of phase transitions, but first I will distinguish between different kinds of inter-theory reduction. 


\subsection{Types of Intertheory Reduction: Reduction ${ }_{1}$, Reduction Re $_{2}$ and Limiting reduction}

In his influential paper "Two Concepts of Intertheoretic Reduction" (1973), Nickles distinguishes between what he calls reduction ${ }_{1}$ and reduction . $_{\text {. }}$

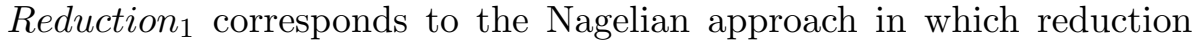
is essentially understood in terms of logical deduction (derivability). In its most strict version, this approach requires the reduced theory $T_{h}$ to be a logical consequence of $T_{l}$ plus bridge laws, which have the function of connecting the vocabulary of $T_{h}$ with the vocabulary of $T_{l}$ (connectability). A general formulation of this model is as follows:

Reduction $_{1}: T_{h}$ reduces $_{1}$ to $T_{l}$ iff the laws of $T_{h}$ can be logically deduced from the laws of $T_{l}$ along with bridge laws that connect the terms of $T_{l}$ and $T_{h}$.

Inspired by cases in which the deduction of the higher level theory from the lower level theory is not exact, Schaffner (1967) and Nagel himself (1970) suggest the following revision of the Nagelian model or reduction 1 (sometimes called the Generalized Nagel Schaffner model, for example in DizadjiBahmani, Frigg, and Hartmann 2010):

Reduction* $: T_{h}$ reduces $s_{1}^{*}$ to $T_{l}$ iff there is a corrected version $T_{h}^{*}$ of $T_{h}$ such that (i) the laws of $T_{h}^{*}$ can be logically deduced from the laws of $T_{l}$ along with bridge laws and (ii) $T_{h}$ and $T_{h}^{*}$ are 'strongly analogous'.

It is clear that in cases of reduction 1 in which the higher level theory is proved to be a logical consequence of the lower level theory, one achieves domain subsumption in the sense described in Sec. 3.1. ${ }^{7}$ What is less clear is whether the same goal can be achieved by reduction. ${ }_{1}^{*}$. Since domain subsumption requires that the low level theory $T_{l}$ accounts for the behavior that is successfully described by the original higher level theory $T_{h}$ and not by the corrected theory $T_{h}^{*}$, the success of this approach to reduction will crucially depend on the demonstration that there is indeed a strong analogy relationship between $T_{h}$ and $T_{h}^{*}$. Although there is no general characterization of "strong analogy", in most cases this will signify that the two theories are in close agreement or approximately equal, for example, with respect to the predictions that they make. Whether or not there is an approximation relation between $T_{h}$ and $T_{h}^{*}$ will depend on empirical considerations and will be decided within the specific scientific discipline (Rosaler 2017, DizadjiBahmani et al. 2010). Important for Reduction* is that the approximation

\footnotetext{
${ }^{7}$ There is an important controversy about the status of the bridge laws in reduction 1 that I will not address here. For a detailed discussion on this issue see, for example, Dizadji-Bahmani et al. 2010
} 
relation should warrant that the corrected theory $T_{h}^{*}$ can account (at least approximately) for the behavior that is well described by $T_{h}$.

A second type of reduction distinguished by Nickles is reduction ${ }_{2}$ that basically consists in the recovery of one theory from another by applying limiting operations or other appropriate transformations. ${ }^{8}$ In contrast to reduction $_{1}$, reduction 2 is not restricted to a single logical relation but can involve a series of intertheoretic operations. More importantly, in reduction 2 the derivation of a theory from another should be understood in a broad sense including not only logical deduction but also limiting operations and approximations of many kinds. So, in contrast to reduction 1 and also to reduction $_{1}^{*}$, in reduction 2 logical deduction may not play any role at all. Nickles (1973) provides the following characterization of reduction ${ }_{2}$ :

Reduction ${ }_{2}$ : Let $O_{i}$ be a set of intertheoretic operations, then a theory $T_{h}$ reduces $_{2}$ to another $T_{l}$ iff $O_{i}\left(T_{l}\right) \rightarrow T_{h}$, where the arrow represents "mathematical derivation" understood in a broad sense including not only logical deduction but also limiting operations and approximations of many kinds. ${ }^{9}$

Roughly, this schema signifies that by performing a set of mathematical operations $O_{i}$ on $T_{l}$ one obtains $T_{h}$.

Now, one should note that mathematical operations such as limits and other approximations are performed not on the theory itself but on functions (or equations) representing physical quantities. Therefore a more precise schema needs to be formulated in terms of the relevant quantities and not directly on the theories to be compared. Moreover, if one wants reduction 2 to assure that the lower level theory $T_{l}$ can account for the behavior that is well described by the higher level theory $T_{h}$, one should add informal or empirical constraints. ${ }^{10}$ As Nickles himself recognizes, in order for reduction ${ }_{2}$ to hold, it is necessary that the mathematical operations performed on $T_{l}$ make physical sense. Although he is not explicit about what he means by

\footnotetext{
${ }^{8}$ Some philosophers (e.g. Norton 2012, Batterman 2005) have referred to reduction 2 as the physicist' sense of reduction and to reduction r $_{1}$ as the philosophers' sense of reduction. This distinction is motivated by the fact that philosophers generally speak of a less fundamental theory reducing to a more fundamental one, whereas physicists tend to invert this concept of reduction by saying that it is the more fundamental theory which reduces to a less fundamental one under limiting operations or other transformations. Since I believe that both types of reduction can serve to philosophical goals such as explanation and ontological economy, I will not draw this distinction here.

${ }^{9}$ For Nickles, in reduction 2 one says that the more fundamental theory reduces to the less fundamental one under certain mathematical operations. This inversion in the direction of the reduction is motivated by the way in which physicists generally talk about reduction. Since this is not important to understand this approach to reduction, I will stick to the philosophers' jargon.

${ }^{10}$ See Rosaler 2017 for a discussion of the importance of including empirical criteria in the different approaches to reduction.
} 
"physical sense", one can interpret this constraint as signifying that after applying a set of mathematical operations on $T_{l}$, the resulting theory $T_{l}^{*}$ can still describe realistic behavior. Taking the limit of a constant of nature to zero, for example, may result in a theory that does not account for realistic behavior unless this limit is adequately explained. Similarly taking the limit of a parameter such as temperature or the number of particles to infinity may also be illegitimate if these limits are not adequately justified. I will come back to this point below when I address limiting reduction.

The previous considerations lead to a revised formulation of reduction 2 that can be expressed as follows:

Reduction ${ }_{2}^{*}$ : Given a set of intertheoretic operations $O_{i}$, a quantity $Q^{l}$ of $T_{l}$ reduces $s_{2}^{*}$ a quantity $Q^{h}$ of $T_{h}$ iff (i) $O_{i}\left(Q^{l}\right)=Q^{h}$ and (ii) the mathematical operations $O_{i}$ make physical sense.

Naturally, in order to obtain the reduction of one theory to another, one would require that all the physically relevant quantities of $T_{h}$ reduce $e_{2}^{*}$ to the quantities of $T_{l}$ under certain conditions. The choice of relevant quantities will depend on the case study and will be determined by empirical considerations. Proving that all relevant quantities in a theory reduce to the corresponding quantities of another theory is in every case a huge enterprise, but note that, according to the above framework, the failure of reduction of one of the relevant quantities suffices to infer the failure of reduction of an entire theory to another. As it will be seen in the next section, this is exactly what is at stake in the case of phase transitions.

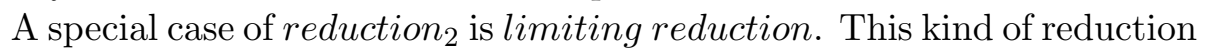
is frequently equated to reduction ${ }_{2}$, but it is important to distinguish between these two cases. In fact, whereas reduction 2 does not specify the kind of mathematical transformations that are applied on $T_{l}$, limiting reduction refers to cases in which the transformations consist in mathematical limits. In cases where one limit is applied, one can express limiting reduction as follows:

Limiting reduction: Let $Q^{l}$ denote a relevant quantity of $T_{l}$, $Q^{h}$ a relevant quantity of $T_{h}$, then a quantity $Q^{h}$ of $T_{h}$ limiting reduces to a corresponding quantity $Q^{l}$ of $T_{l}$ iff (i) $\lim _{x \rightarrow \infty} Q_{x}{ }^{l}=$ $Q^{h}$ (where x represents a parameter appearing in $T_{l}$ ) and (ii) the limiting operation makes physical sense.

The requirement that the limiting operation makes physical sense is related with the justification of mathematical limits. As Menon and Callender (2013, 201) puts it:

One might not think that a fully reductive explanation has been given unless one can explain using the resources of the reducing theory why this model is so effective under those conditions. 
Why does modeling a finite particle phase transition as nonanalytic work so well at the thermodynamic level of description if finite systems cannot exhibit non-analyticities at the statistical mechanical level of description? If we cannot give such an explanation, we have another potential variety of emergence: explanatory irreducibility.

For Menon and Callender, the justification of infinite idealizations is related with the possibility of de-idealizing the model that uses infinite idealization or, in other words, with the possibility of showing that the behavior described by infinite systems is well approximated by a finite system. In a similar vein, Butterfield (2011) suggests a criterion for the justification of infinite limits (called by Landsman (2013) "The Butterfield Principle") according to which a limit is justified as being mathematically convenient and empirically adequate, if the values of the quantities evaluated in the limit at least approximate the values of the quantities "on the way to the limit", i.e., for large but finite values of the parameter $x_{0}: \lim _{x \rightarrow \infty} \mathrm{Q}_{x} \approx Q_{x_{0}}$. In addition, Palacios 2018 points out that it does not suffice to show that the behavior that arises in the limit arises "on the way to the limit", but one also needs to show that it arises for realistic values of $x$. This latter requirement ought to be empirically grounded and is meant to assure that the lower level theory that results from a limiting operation is empirically correct and therefore capable of describing realistic behavior.

\subsection{Reduction R $_{1}$ and Limiting Reduction Combined in the Case of Phase Transitions}

Now that we have distinguished between different approaches to reduction, we are in a position to evaluate which one is the most suitable for describing the case of phase transitions. As said above, most philosophers (Butterfield 2011, Butterfield and Bouatta 2011, Norton 2012) advocating a reductionist position on phase transitions have argued that this is a case of Nagelian reduction or what has been called here "reduction 1 ". In particular, Butterfield (2011) and Butterfield and Bouatta (2011) suggest that phase transitions combine reduction ${ }_{1}$ and emergence. In their view, reduction ${ }_{1}$ describes the relationship between thermodynamics $T D$ taken as the higher level theory and statistical mechanics in the thermodynamic limit $S M^{\infty}$, which describes a system with " $N=\infty$ ". They claim that since the exact values of the quantities that describe phase transitions in $T D$, i.e. derivatives of the free energy, can be recovered from $S M^{\infty}$, one can deduce the thermodynamic treatment of phase transitions from the latter theory by defining appropriate bridge laws. On the other hand, they also argue that the behavior that arises in the limit, i.e. singularities in the derivatives of the free energy, is "emergent" in the sense of being "novel and robust" 
with respect to the behavior described by finite statistical mechanics $S M$, because - as it was seen in Sec. 2 - strictly speaking the singularities cannot be derived for finite $N$. Interestingly, they maintain that this notion of emergence is "weak" in the sense that approximately the same behavior that arises in the limit also arises for finite but large values of $N$. This proposal is illustrated in Figure 2.

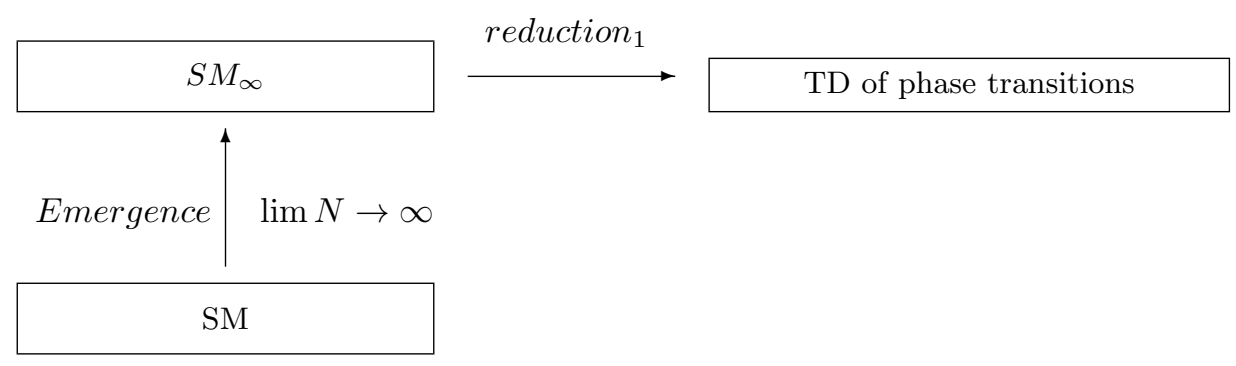

Figure 2: Butterfield-Bouatta schema for phase transitions.

Although Butterfield-Bouatta's proposal is plausible, this weak notion of emergence appears to be an artifact of sticking to a definition of reduction that is far too strict. In fact, if it is true that one can recover the behavior observed in the limit from finite statistical mechanics for large but finite values of $N$, why should we talk about emergence at all in this context? In fact, we have argued in the previous section that the goal of reduction was to demonstrate that the behavior that can be adequately described by the high level theory can be also described by the low level theory; therefore if finite statistical mechanics can account, at least approximately, for the behavior described by thermodynamics, then we would have a case of reduction simpliciter. The caveat here is that the intended reductive relationship between thermodynamics and finite statistical mechanics cannot be described just by reduction $_{1}$ (i.e. Nagelian reduction), since the singularities that successfully describe phase transitions in thermodynamics cannot be logically deduced from finite statistical mechanics. Instead, the appropriate model of reduction should allow for limiting operations and other approximations that do not demand a theory to be embedded into the other. More specifically, given that the recovery of the quantities that describe phase transitions in thermodynamics involves first taking the thermodynamic limit in statistical mechanics and then defining bridge laws to relate the relevant quantities in both theories, this is a case that (potentially) combines limiting reduction

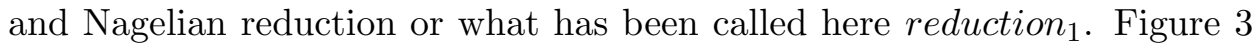
illustrates how this reductive relation would look for both first order and 
continuous phase transitions. Whether or not this reductive framework succeeds will be addressed in the next section.

\section{First order phase transitions}

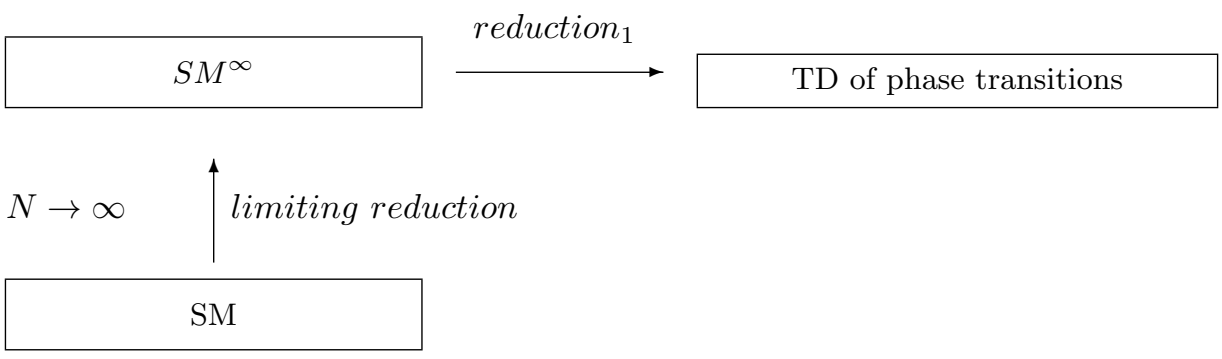

\section{Continuous phase transitions}

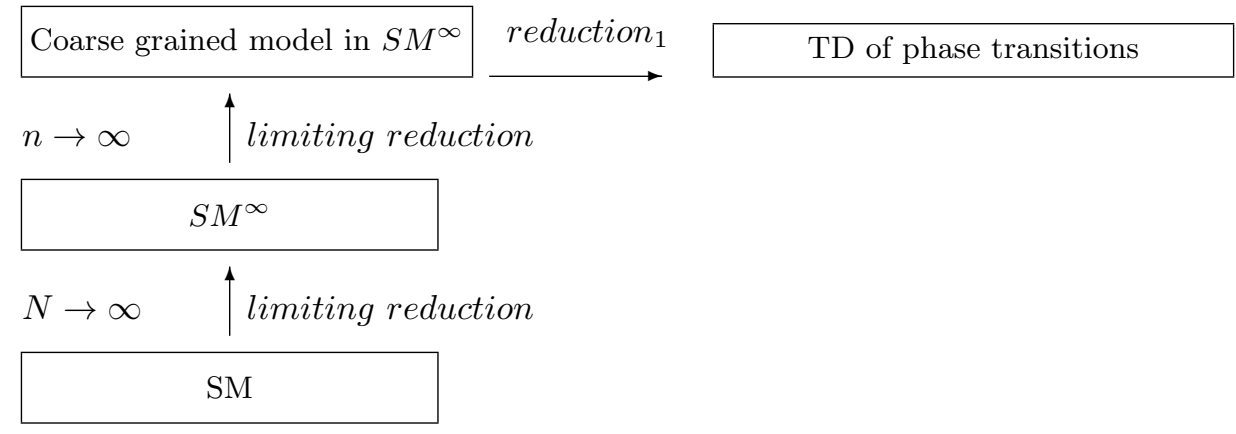

Figure 3: Candidate schema for the reduction of first order and continuous phase transitions.

Nagelians may say that this schema fits well with reduction* (or the GNS-model) and that "limiting reduction" just proves that there is a strong analogy between $S M$ and $S M^{\infty}$. Although one can always adapt the Nagelian model to cover this kind of cases, the usual characterization of reduction* does not provide us with a natural framework for cases like phase transitions. As we saw above, in reduction $n_{1}^{*}$, logical deduction describes the relationship between the low level theory $T_{l}$ and a high level theory $T_{h}^{*}$, which is a corrected version of the original theory $T_{h}$, and the notion of "strong analogy" describes the relationship between the original secondary theory $T_{h}$ and the corrected version $T_{h}^{*}$. The prototypical example for this kind of reduction is the Second Law of thermodynamics, in which it is argued that there is a strong analogy between the exact Second Law and the theory that can be actually deduced from statistical mechanics, which postulates that entropy fluctuates in equilibrium.

There are two important differences between the derivation of the Second 
Law and the usual derivation of the thermodynamic theory phase transitions from statistical mechanics. First, in the case of phase transitions one does not derive a corrected version $T_{h}^{*}$ of the thermodynamic treatment of phase transitions $T_{h}$. Instead, with the help of bridge laws, one derives the exact thermodynamic treatment of the phenomena $T_{h}$, in which phase transitions are defined in terms of singularities in the derivatives of the free energy. Second, the derivation of $T_{h}$ from $T_{l}$ should not be interpreted as logical deduction since there are limiting operations involved that do not preserve the content of the fundamental theory. Agreed, Nagelians may still argue that this is a case of approximative or imperfect reduction ${ }_{1}$ in which the derivation of the secondary theory is not exact. Nevertheless, I believe that this strategy would be misleading. In fact, if we accept that the goal of reduction is the subsumption of the domain of applicability of a theory by another, we should not argue that the use of these limiting operations leads to an approximative or imperfect reduction, but rather to a different kind of reduction that does not require a theory to be a logical consequence of the other. Nickles (1973) have presented an alternative model of reduction, which is meant to describe cases, in which limits occupy a central role in the derivation of the high level quantities. The limitation of Nickles' account is that he restricted limiting reduction to cases of homogenous reduction that do not require the use of bridge laws. The need for bridge laws in the derivation of phase transitions means that this case cannot be entirely covered by Nickles' limiting reduction. Instead, it is a combination of Nickles' model of reduction and Nagelian reduction that provides an adequate candidate framework for the reduction of phase transitions.

We have argued in this section that the candidate schema for the reduction of phase transitions is a combination of reduction ${ }_{1}$ and limiting reduction. In the next section we will evaluate whether the proposed schema actually holds for both first order and continuous phase transitions.

\section{Are Phase Transitions Incompatible with Re- duction?}

In order to judge whether phase transitions correspond to a case of reduction according to the schema presented in the previous section, one needs to specify which quantities of $S M$ and $T D$ are expected to display the same behavior. Based on what has been said in Section 2.2, one can observe that in the case of first-order phase transitions one is interested in recovering from statistical mechanics the singularities in the derivatives of the free energy that successfully describe the phenomenon in thermodynamics. On the other hand, in the case of continuous phase transitions one is not interested in computing the free energy but rather in calculating the universal quantities that control the behavior of the thermodynamic quantities, e.g. $M, C, \chi$, 
close to the critical point, in particular one is interested in calculating the value of the critical exponents $\alpha, \beta, \gamma$.

For the case of first-order phase transitions, the singularities in the derivatives of the free energy are successfully obtained in the limit theory $S M^{\infty}$, which allows one to construct bridge laws that relate the thermodynamic derivatives of the free energy $F^{T D}$ with the corresponding quantities in "infinite statistical mechanics" $F^{S M^{\infty}}$. For the case of continuous phase transitions, the correct values of the critical exponents are usually obtained by taking a second infinite limit (iteration limit $n \rightarrow \infty$ ) in infinite statistical mechanics, which allows one to construct bridge laws that relate the thermodynamic quantities $T D$ with the quantities defined in infinite statistical mechanics $S M_{\infty}$. The problem is that in order to reduce thermodynamic behavior of phase transitions to statistical mechanics, one should relate the behavior of the thermodynamic quantities not only with statistical mechanics in the limit $S M^{\infty}$, but also with finite statistical mechanics $S M$. This implies that we need to show that there is a limiting reduction between the quantities evaluated in the limit system and the quantities evaluated in systems with finite and realistic values of the parameters that go to infinity. Although Batterman and other authors have contended that this cannot be done, and therefore, that phase transitions are not reducible to statistical mechanics, I will argue next that there is indeed limiting reduction in both first-order and continuous phase transitions.

\subsection{The Problem of "Singular" Limits and Butterfield's So- lution for First Order Phase Transitions}

The view that limiting reduction fails in the case of phase transitions has been most notably developed by Batterman (2001; 2005; 2011), who argues that this is a consequence of the "singular" nature of the thermodynamic limit. $^{11}$

Using Batterman's terminology, a limit is singular "if the behavior in the limit is of a fundamentally different character than the nearby solutions one obtains as $\epsilon \rightarrow 0$ " (Batterman, 2005, p. 226). According to him, the thermodynamic limit is singular in this sense because no matter how large we take the number of particles $N$ to be, as long as the system is finite, the derivatives of the free energy will never display a singularity. As a consequence, he says that finite statistical mechanics $S M$ does not allow us to construct a model or theory that approximates the thermodynamic behavior of phase transitions and that it is only the limit theory $S M^{\infty}$, for which $N=\infty$, that can provide such an account.

The idea that we can find analytic partition functions that "approximate" singularities is mistaken, because the very notion of

\footnotetext{
${ }^{11}$ Similar views are also held by Rueger (2000) and Morrison (2012).
} 
approximation required fails to make sense when the limit is singular. The behavior at the limit (the physical discontinuity, the phase transition) is qualitatively different from the limiting behavior as that limit is approached (Batterman, 2005, p. 236).

Based on what has been stated in Section 3, this would mean the values of the quantities evaluated in the limit theory $S M^{\infty}$ do not coincide with the limit of values of the quantities obtained in the finite theory as $N$ tends to infinity and that the first condition for limiting reduction fails:

$$
\lim _{N \rightarrow \infty} Q_{N}^{S M} \neq Q^{S M_{\infty}} .
$$

Although, it is true that the singularities in the derivatives of the free energy can only be obtained in the limit $N=\infty$, Butterfield (2011) (and Butterfield and Bouatta (2011)) correctly points out that this does not mean that finite statistical mechanics cannot approximate the values obtained in the limit $N=\infty$. He illustrates this point with the following mathematical example. Consider the following sequence of functions:

$$
g_{N}(x)= \begin{cases}-1 & \text { if } x \leq-1 / N \\ N_{x} & \text { if }-1 / N \leq x \leq 1 / N) \\ 1 & \text { if } x \geq 1 / N\end{cases}
$$

As $N$ goes to infinity, the sequence converges pointwise to the discontinuous function:

$$
g_{\infty}(x)= \begin{cases}-1 & \text { if } x<0 \\ 0 & \text { if } x=0 \\ 1 & \text { if } x>0\end{cases}
$$

One can introduce another function $f$, such that

$$
f= \begin{cases}1 & \text { if } g \text { is continuous } \\ 0 & \text { if } g \text { is discontinuous }\end{cases}
$$

If one focuses on $f$, then one may conclude, in the same vein as Batterman, that the value of $f_{\infty}$ at the limit $N=\infty$ is fundamentally different from the value when $N$ is arbitrarily large but finite and that limiting reduction fails. However, Butterfield warns us that if we look at the behavior of the function $g$, we will see that the limit value of the function is approached continuously and therefore that the limit system is not "singular" in the previous sense, so that $\lim _{N \rightarrow \infty} g_{N}=g_{\infty}$ holds.

According to Butterfield, this is exactly what happens with classical phase transitions and, for the case of first-order phase transitions, he seems 
right. ${ }^{12}$ In fact, in a system containing a finite number of particles and confined to a restricted geometry, the thermodynamic quantities that describe first-order phase transitions such as density and magnetization are analytic and vary smoothly with the relevant parameters of the problem. Therefore, if we introduce a quantity $H$ representing the discontinuity of such quantities and attribute a value 1 to $\mathrm{H}$ if the quantity is discontinuous and 0 if it does not (analogously to the function $f$ in Butterfield's example), then we might conclude that such a quantity has values evaluated at the limit $N=\infty$ that are considerably different from the values of the of systems "on the way to the limit", i.e. for finite $N$. However, if we focus on the behavior of the thermodynamic quantities themselves, namely density for the liquid-gas transition and magnetization for the change of magnetization of a ferromagnet at sub-critical temperatures, we will arrive at a different conclusion. In fact, as $N$ grows, the change in the thermodynamic quantities becomes steeper and steeper so that the quantities smoothly approach a discontinuity in the limit (analogous to the function $g$ ). More importantly, a theoretical analysis of those situations also show that these limits make physical sense, since one can demonstrate that for realistic values of $N$, the gradient in the derivatives of the free energy $F^{S M}$ is sufficiently steep so that the difference in the limit values of the thermodynamic quantities as $N \rightarrow \infty$ and realistic systems with finite $N_{0}$ becomes negligibly small: $\lim _{N \rightarrow \infty} F_{N}^{S M} \approx F_{N_{0}}^{S M}$. (Schmelzer and Ulbricht 1987, Fisher and Berker 1982). As Kadanoff (2009, p. 783) puts it:

As the number of lattice sites gets larger the variation in the magnetization will get steeper, until at a very large number of sites the transition [...] will become so steep that the casual observer might say that it has occurred suddenly. The astute observer will look more closely, see that there is a very steep rise, and perhaps conclude that the discontinuous jump only occurs in the infinite system.

The important lesson from Butterfield's argument is that the "singular" nature of the thermodynamic limit does not imply that there are no models of statistical mechanics that approximate the thermodynamic behavior of phase transitions, for $N$ sufficiently large but finite. For him, this should be taken as supporting the claim that the discontinuities that describe phase transitions in the limit are not physically real, but rather that they approximate the behavior of realistic systems. For us, this should be taken as an

\footnotetext{
${ }^{12}$ Even if Butterfield aims to make a more general claim, this does not hold for all cases of "singular" limits. Landsman (2013) shows that for the case of quantum systems displaying spontaneous symmetry breaking and the classical limit $\hbar \rightarrow 0$ of quantum mechanics, the situation is different and much more challenging. It seems therefore that the analysis of singular limits and the way of "dissolving the mystery" around them should be done on a case-by-case basis.
} 
argument in favor of the existence of limiting reduction between $S M$ and $S M^{\infty}$

One needs to be cautious, however, in not concluding that the previous argument solves all the controversy around the reduction of phase transitions. First of all, it is important to bear in mind that we are referring only to classical phase transitions and that quantum phase transitions have not been considered. ${ }^{13}$ Second, one needs to note that we have not considered the use of renormalization group methods yet, in which there are two infinite limits involved. This is precisely the issue that we are going to address next.

\subsection{Dissolving the Problem of Singular Limits for Continu- ous Phase Transitions}

As was shown in Section 2, the inference of the thermodynamic behavior of continuous phase transitions generally requires the appeal to RG methods. Batterman (2017) has suggested that the assumption of RG methods poses a further challenge for the project of reducing phase transitions to statistical mechanics. He attributes this difficulty to the need for the thermodynamic limit in the inference of fixed point solutions, which are said to be necessary for the computation of critical exponents and for giving an account of universality. He claims (Batterman 2017, p. 571):

Crucial, of course, to finding a fixed point, is the fact that at criticality the correlation length diverges. Unless one has correlations of infinite extent, the iterative blocking procedure will get hung up at a given length scale and one will not find the fixed point.

Batterman associates the essential role played by the correlation length with the singularity of the thermodynamic limit. However, what he overlooks is that in continuous phase transitions there is a second infinite limit $n \rightarrow \infty$ involved, which corresponds to iterating infinitely many times the RG transformations. Realizing that there is a second limiting operation is important because it allows us to see that the set of mathematical limits involved in the statistical mechanical treatment of continuous phase transitions is not singular in Batterman's sense. In fact, once the thermodynamic limit is taken, the infinite iteration limit approaches smoothly the non-trivial fixed $\overline{0}$ ations $K$ that allow one to calculate the values of the critical exponents, so that the following expression holds: $\lim _{n \rightarrow \infty} \lim _{N \rightarrow \infty} K_{N, n}^{S M}=K^{S M_{\infty}}$. This is a consequence of the analyticity of the renormalization group flow, which is a basic assumption of the $\mathrm{RG}$ framework. The problem is rather that these two limits do not commute since for large but finite values of $N$, the second infinite limit $n \rightarrow \infty$

\footnotetext{
${ }^{13}$ For an analysis of quantum phase transitions see Landsman (2013).
} 
leads to trivial fixed points $K^{*}$ that do not allow one to calculate the correct values of the critical exponents. In other words, this means that for large but finite $N: \lim _{n \rightarrow \infty} K_{n}^{S M} \neq K^{S M_{\infty}}$. The reason for this result is - as we saw in Sec. 2 - that in every finite system there will be a characteristic length scale associated to the size of the system. Therefore, the application of a coarse-graining transformation beyond that length will no longer give identical physical systems and the "RG flow" will inevitably move towards a trivial fixed point, with values of the coupling constants either $K=0$ or $K=\infty$.

If we recall the conditions for limiting reduction mentioned in section 3 , we will see, however, that the previous result does not imply a failure of limiting reduction between the quantities evaluated in finite statistical mechanics $S M$ and the limit theory $S M^{\infty}$ for which $N=\infty$ and $n=\infty$. In fact, limiting reduction requires (i) that the quantities evaluated in the limit theory can be recovered by performing a set of limiting operations on the low level theory, and (ii) that these limiting operations make physical sense. We have seen here that the values of the critical exponents can be actually recovered by the set of infinite limits performed on statistical mechanics and that the set of limiting operations is not singular so that condition (i) holds. In the next section, we will see that the mathematical limits involved in the treatment of continuous phase transitions also make physical sense.

\subsection{Justifying the thermodynamic limit and the infinite it- eration limit for Continuous Phase Transitions}

Since the goal of limiting reduction is to demonstrate that the lower level theory can account for the behavior that is well described by the high level theory, the limiting operations should make physical sense. This means that as $N \rightarrow \infty$ and $n \rightarrow \infty$ the quantities should still be able to describe the behavior of realistic systems. In the case of first-order phase transitions, where there are no renormalization group methods involved, the relevant quantities that describe a phase transitions, such as density, approximate the behavior of the quantities evaluated in the limit not only for large and finite values of $N$, but also for realistic values of the parameter. The challenge for the reductionist is to show that the two limiting operations involved in the statistical mechanical treatment of continuous phase transitions are also physically meaningful in the previous sense, in other words, that the fixed point solutions obtained by taking two infinite limits can be also obtained, at least approximately, in systems with finite values of $N$. Even philosophers with a reductionist bent towards phase transitions are still skeptical about the possibility of approximating non-trivial points in finite systems. Menon and Callender (2013, p. 219), who endorse in general the reducibility of phase transitions, say for instance: 
There does not seem to be a clear sense in which the renormalization flow of finite systems can approximate a fixed point. A point is either a fixed point for the flow or it isn't; it can't be "almost" a fixed point. And unlike Butterfield's example, there does not seem to be a way of re-phrasing the explanation of universality in terms that are approximated by large finite systems.

The problem, as we saw, is that when we apply the infinite iteration limit $n \rightarrow \infty$ in a system with finite $N$ and finite correlation length, the system will approach trivial fixed points that do not allow one to calculate the values of the critical exponents that can be successfully calculated when the two limits are performed. The reason for this is that the two limits do not commute. Indeed, if one takes the infinite iteration limit after the thermodynamic limit, one obtains the correct results, but if one takes the infinite iteration limit before taking the thermodynamic limit, i.e. in a finite system, one ends up in a trivial fixed point that do not allow one to compute the correct values of the critical exponents. It appears, therefore, that the values of the quantities evaluated in the limits $N=\infty$ and $n=\infty$ do not approximate the values of the quantities evaluated in realistic systems with finite $N$.

What the reductionist needs to realize, however, is that the infinite iteration limit is needed only if we consider an infinite system. If we consider a finite system, there is no need to take the infinite iteration limit. Furthermore, one should not take the infinite iteration limit. In fact, if one could demonstrate that the limit values of the quantities as $N \rightarrow \infty$ and $n \rightarrow \infty$ can be approximated by the quantities evaluated in finite system with realistic value of $N_{0}$ and "before" we get to the infinite iteration limit, i.e. for finite values $n_{0}$, one would have every reason to conclude that the infinite limits are physically significant and, therefore, that this ase of limiting reduction. Batterman (2017) seems to recognize this poi out he is skeptical about the possibility of finding fixed points after a finite number of iterations. In a footnote (p. 571), he writes:

It seems to me that if one is going to hold that the use of the infinite limits is a convenience, then one should be able to say how (even if inconveniently) one might go about finding a fixed point of the RG transformation without infinite iterations. I have not seen any sketch of how this is to be done. The point is that the fixed point, as just noted, determines the behavior of the flow in its neighborhood. If we want to explain the universal behavior of finite but large systems using the RG, then we need to find a fixed point and, to my knowledge, this requires an infinite system.

Fortunately for the reductionist, there are topological and numerical 
arguments supporting the possibility of calculating non-trivial fixed points in finite systems. In order to understand those arguments, one needs to study first the topology of the renormalization group flows. Figure $1=$ escribes a contour map sketching such a topology and serves to illustrate the situation. Here the RG flows are represented by the trajectories $R$ and $D$ in a space $S$ of Hamiltonians. Each point in this space represents a physical system described by a particular Hamiltonian associated with a set of coupling constants $K$. In this topology, the elements of $S$ can be classified according to their correlation lengths $\xi$. Therefore, one can define surfaces containing all Hamiltonians $H \in S$ with a given correlation length. The critical surface describes the set of all Hamiltonians with infinite correlation length $\xi=\infty$. In the figure, $p$ represents a system with a Hamiltonian that inhabits the critical surface $\xi=\infty$, whereas $s$ represents a system with a Hamiltonian that is infinitesimally close to $p$ but is not on the critical surface; $p^{*}$ and $p_{0}$ are fixed points. As one can see, the trajectory $D$ starting from $s$ will stay close to trajectory $\mathrm{R}$, describing a system at criticality, but eventually will move away towards a trivial fixed point $p_{0}$. This follows because in a finite system the RG transformation will constantly reduce the value of the correlation length, moving the system away from criticality and resulting in a system with trivial values of coupling constants. Since the values of the critical exponents can be calculated by linearizing around non-trivial fixed points, this naturally means that iterating the RG transformation infinitely many times in a finite system will lead us to a fixed point from which one will be able neither to compute the critical exponents nor to give an account of universality.

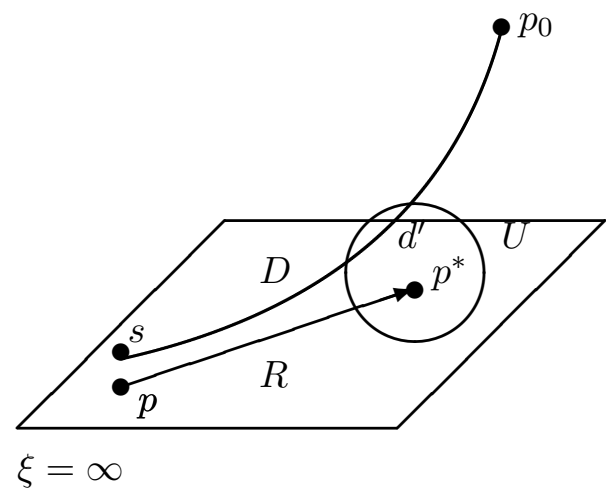

Figure 4: Contour map sketching the topology of the renormalization group flow (R). $s$ and $p$ represent systems infinitesimally close to each other. $p^{*}$ is a critical fixed point and $p_{0}$ is a trivial-fixed point. The region around the fixed point $p^{*}$ represents neighboring points. 
Despite the previous result, Wilson and Kogut (1974, Sec. 12) demonstrated by using $\epsilon$-expansion approximation that in principle, and for an idealized case, if one starts from a point which is close enough to the critical surface, represented by " $s$ " in Figure 3 th e RG trajectory $D$ will move close to the critical trajectory until it reaches the vicinity $U$ of a non-trivial fixed point $p^{*} \cdot{ }^{14}$ Once the trajectory reaches the neighborhood $U$ of the fixed point $p^{*}$ will stay there for a long time (which means, for repeated iterations of the RG transformation), thereby acting as it were a fixed-point $d^{\prime}$. Finally, as $n \rightarrow \infty$, the trajectory will eventually move away from that region approaching a trivial fixed point. What is relevant for us is that within the neighborhood $U$ and from an "effective" fixed point $d^{\prime}$ linearization is indeed possible and allows for the calculation of accurate values of the critical exponents. The latter implies that the values of the critical exponents evaluated in a finite system after a finite number of iteration $n_{0}$ can in principle approximate the values of the critical exponents obtained after taking the limits $N \rightarrow \infty$ and $n \rightarrow \infty$ so that the behavior that arises in the limit is approximated by the behavior for large but finite values of $N$ and $n$ :

$$
K_{N_{0}, n_{0}}^{S M} \approx \lim _{n \rightarrow \infty} \lim _{N \rightarrow \infty} K_{N, n}^{S M}
$$

Yin (2011), based on previous work done by Haller and Kennedy (1996), reinforces this conclusion by deriving an upper bound for the linearization in the neighborhood of non-trivial fixed points, which helps estimate the error associated with the linear approximation. It is important to note, however, that in order to derive accurate values of the critical exponents, the number of iterations of the RG transformations should be large enough so that all details which are not universal, namely all details specific to a model, are washed out. If the number of iterations is not large enough the coupling constants will be sensitive to details of the model and the calculations of critical exponents will not be accurate (For details see also Le Bellac et. al 1998).

One might object that the previous results rely too much on an idealized case and they do not serve to describe realistic situations. Although it is true that in practice things are less straightforward, Monte Carlo simulation gives an important support for the claim that finite systems with more realistic values of $N$ will display the desired behavior. Since 1976 there have

\footnotetext{
${ }^{14}$ The $\epsilon$-expansion is an asymptotic expansion for which $\epsilon$ takes values from $\epsilon=1$ to $\epsilon<<1$. Since the exponents are not analytic at $\epsilon=0$ one faces convergence problems which are treated by sophisticated summation methods that are nowadays under control.
} 
been attempts to use the numerical Monte Carlo simulation in the framework of renormalization group methods for the study of critical exponents. The first contribution in this direction was made by Ma (1976), who suggested an application of real space RG methods that required the calculation of the renormalized Hamiltonians. However, since calculating the renormalized couplings accurately enough proved to be too difficult, this approach did not succeed in determining the fixed point Hamiltonian with significant precision. Pawley, Swendsen, and Wilson (1984) made further progress in this direction by suggesting an approach based on expectation values of the correlation functions that did not rely on the calculation of renormalized Hamiltonians. Using this approach, they showed that for an Ising square lattice with 64 number sites, the system approaches the behavior of an infinite system after two iterations of a RG transformation. After more iterations, however, the system was shown to depart from the expected results flowing towards a trivial fixed point. A plausible explanation for this cross-over was that after more iterations the correlation length became comparable to the size of the system and finitary effects became relevant. Mainwood (2006), Butterfield (2011) and Menon and Callender (2011) stress the possibility of cross-over but since they did not offer a topological explanation of the two infinite limits involved in the treatment of continuous phase transitions, they did not succeed in giving a rebuttal argument for the reduction of continuous phase transitions. On the other hand, the arguments presented here give us good reason to conclude that the behavior derived in the limits $N \rightarrow \infty$ and $n \rightarrow \infty$ can be approximated by the behavior "on the way to the limit" and, moreover, for realistic values of $N$. If the ultimate goal of inter-theory reduction is to demonstrate that the low level theory can account for the behavior that is successfully described by the high level theory, then, based on the previous arguments, we have all reasons to conclude that the thermodynamics of phase transitions reduces to statistical mechanics.

\subsection{On the Indispensability of Infinite Limits}

In this section we have addressed the issue of whether phase transitions reduce to statistical mechanics. A different issue that has been at stake in the philosophical literature is whether the infinite limit is "essential" to give an account of phase transitions. Although this is not the topic of this paper, I will allow myself a short note on this matter. Here we have said that the behavior of the quantities that successfully account for phase transitions in the limits $N$ and $n$ approximate the behavior of the corresponding quantities evaluated in systems with finite values of $N$ and $n$. This might well lead one to the conclusion that the infinite limits invoked in the theory of phase transitions are not essential and that it would be possible to construct a finite theory of phase transitions that do not invoke infinite limits at all. Even if I believe that one should not rule out the possibility of constructing such a 
theory - in fact there have been attempts to do so (e.g. Gross 2001, Casetti and Kastner 2006, Borrmann, Mülken, and Harting 2000) -, it is important to emphasize some epistemic roles of the infinite limits. ${ }^{15}$. First of all, the appeal to infinite idealizations makes calculations more tractable. Indeed, it is generally easier to work with infinite sums rather than with large but finite sums. Second, and more importantly, it allows us to remove irrelevant details, such as finitary effects. In fact, in any finite system the finitary or edge effects become relevant when the correlation length approaches the size of the system. Finally, infinite idealizations allow us to provide more rigorous definitions for phase transitions. As we saw previously, the concept of non-analyticity and scale invariance is well-defined only in the limit. ${ }^{16}$

\section{Concluding Remarks}

The arguments presented in this paper give us good reason to think that the appeal to the infinite limits in the theory of phase transitions does not represent a challenge for inter-theory reduction. In fact, I have argued that both first-order and continuous phase transitions fit well with a notion of inter-theory reduction that combines limiting reduction and Nagelian reduction.

Nevertheless, this does not mean that phase transitions are not inconsistent with other notions of reduction that have also been discussed in the philosophical literature. Norton (2013), for instance, correctly points out that the case of continuous phase transitions does not satisfy what he calls "few-many reduction", according to which there will be a reduction if the behavior of a system with a few components can be used to explain the behavior of a system with a large number of them. The reason for this is that continuous phase transitions are intrinsically fluctuation phenomena that can only arise when $N$ is sufficiently large. This is certainly an interesting sense in which reduction "fails" for the case of phase transitions, but since traditional statistical mechanics is in general a theory that describes systems with large $\mathrm{N}$, this failure of reduction is not incompatible with the inter-theoretic reduction between thermodynamics and classical statistical mechanics.

Likewise, continuous phase transitions also seem to be at odds with the kind of reductive explanation that requires the explanans to give us accurate and detailed information about the microscopic causal mechanisms that produce the phenomenon (e.g. Kaplan 2011). As has been pointed out by Batterman (2002), Batterman and Rice (2014) and Morrison (2012), the im-

\footnotetext{
${ }^{15}$ For a philosophical discussion on the indispensability of infinite limits see Ardourel (2018)

${ }^{16}$ See Mainwood (2006) for a more detailed description of the roles of the thermodynamic limit.
} 
possibility of giving such an account is related to the robustness of the fixed point solutions under different choices of the initial conditions. This implies that the critical behavior is largely independent of specific microscopic details characterizing the different models and that the statistical mechanical account of phase transitions does not give us complete information about the microscopic mechanisms underlying the transitions. This of course tells us something about the limitations of statistical mechanics, but, again, does not impede the reduction of thermodynamics to statistical mechanics.

\section{Acknowledgments}

I am extremely grateful to Vincent Ardourel, Federico Benitez, Lapo Casetti, Erik Curiel, Benjamin Feintzeig, Sam Fletcher, Roman Frigg, Stephan Hartmann, Karim Thébault, Giovanni Valente, Pauline van Wierst, Jim Weatherall, Charlotte Werndl and Jingyi Wu for helpful discussions and detailed comments on an earlier draft.

\section{References}

Ardourel, V. (2018). The infinite limit as an eliminable approximation for phase transitions. Studies in History and Philosophy of Science Part B: Studies in History and Philosophy of Modern Physics, 62, 71-84.

Bangu, S. (2009). Understanding thermodynamic singularities: Phase transitions, data, and phenomena. Philosophy of Science, 76(4), 488-505.

Bangu, S. (2011). On the role of bridge laws in intertheoretic relations. Philosophy of Science, 78(5), 1108-1119.

Batterman, R. W. (2001). The devil in the details: Asymptotic reasoning in explanation, reduction, and emergence. Oxford University Press.

Batterman, R. W. (2005). Critical phenomena and breaking drops: Infinite idealizations in physics. Studies in History and Philosophy of Science Part B: Studies in History and Modern Physics, 36(2), 225-244.

Batterman, R. W. (2011). Emergence, singularities and symmetry breaking. Foundations of Physics, 41(6), 1031-1050.

Batterman, R. W. (2017). Philosophical implications of kadanoff's work on the renormalization group. Journal of Statistical Physics, 167(3-4), 559-574.

Batterman, R. W., \& Rice, C. (2014). Minimal model explanations. Philosophy of Science, 81(3), 349-376.

Borrmann, P., Mülken, O., \& Harting, J. (2000). Classification of phase transitions in small systems. Physical Review Letters, 84(16), 3511.

Butterfield, J. (2011). Less is different: emergence and reduction reconciled. Foundations of Physics, 41(6), 1065-1135. 
Butterfield, J., \& Bouatta, N. (2011). Emergence and reduction combined in phase transitions. AIP Conference Proceedings 11, 1446(1), 383-403.

Callender, C. (2001). Taking thermodynamics too serously. Studies in History and Philosophy of Science Part B: Studies in History and Philosophy of Modern Physics, 32(4), 539-553.

Casetti, L., \& Kastner, M. (2006). Nonanalyticities of entropy functions of finite and infinite systems. Physical review letters, 97(10), 100602.

Dizadji-Bahmani, F., Frigg, R., \& Hartmann, S. (2010). Who's afraid of nagelian reduction? Erkenntnis, 73(3), 393-412.

Domb, C. (2000). Phase transitions and critical phenomena (Vol. 19). Elsevier.

Fisher, M. E. (1998). Renormalization group theory: Its basis and formulation in statistical physics. Reviews of Modern Physics, $70(2), 653$.

Fisher, M. E., \& Berker, A. N. (1982). Scaling for first-order phase transitions in thermodynamic and finite systems. Physical Review B, 26(5), 2507.

Franklin, A. (2017). On the renormalisation group explanation of universality on the renormalisation group explanation of universality on the renormalization group explanation of universality. Philosophy of Science, 85(2), 225-248.

Goldenfeld, N. (1992). Lectures on phase transitions and the renormalization group. Westview Press.

Gross, D. H. (2001). Microcanonical thermodynamics: Phase transitions in "small" systems. World Scientific.

Haller, K., \& Kennedy, T. (1996). Absence of renormalization group pathologies near the critical temperature. Journal of Statistical Physics, 85(5), 607-637.

Kadanoff, L. P. (2009). More is the same: Phase transitions and mean field theories. Journal of Statistical Physics, 137(5-6), 777.

Kaplan, D. (2011). Explanation and description in computational neuroscience. Synthese, 183(3), 339.

Landsman, N. P. (2013). Spontaneous symmetry breaking in quantum systems: Emergence or reduction? Studies in History and Philosophy of Science Part B: Studies in History and Modern Physics, 44(4), 379-394.

Le Bellac, M., Mortessagne, F., \& Batrouni, G. (2006). Equilibrium and nonequilibrium statistical thermodynamics. Cambridge University Press.

Ma, S.-K. (1976). Renormalization group by monte carlo methods. Physical Review Letters, 37(8)(461).

Mainwood, P. (2006). Phase transitions in finite systems. unpublished MS; (corresponds to Chapter 4 of Mainwood (2006), Available at http://philsci-archive.pitt.edu/8339/.

Menon, T., \& Callender, C. (2013). Ch-ch-changes philosophical questions raised by phase transitions. In R. Batterman (Ed.), The oxford hand- 
book of philosophy of physics. Oxford University Press.

Morrison, M. (2012). Emergent physics and micro-ontology. Philosophy of Science, $79(1), 141-166$.

Nagel, E. (1970). Issues in the logic of reductive explanations. Mind, science and history, 117-37.

Nickles, T. (1973). Two concepts of intertheoretic reduction. The Journal of Philosophy, 70(7)(181-201).

Norton, J. D. (2012). Approximation and idealizations: Why the difference matters. Philosophy of Science, 79(2), 207-232.

Norton, J. D. (2013). Confusions over reduction and emergence in the physics of phase transitions'. available on Norton's website at: http://www.pitt.edu/jdnorton/Goodies/reduction emergence/red em.html.

Palacios, P. (2018). Had we but world enough, and time... but we don't!: Justifying the thermodynamic and infinite-time limits in statistical mechanics. Foundations of Physics, 5(48), 526-541.

Pawley, G., Swendsen, D., R.H. Wallace, \& Wilson, K. G. (1984). Monte carlo renormalization-group calculations of critical behavior in the simple-cubic ising model. Physical Review, 29(7), 4030.

Rosaler, J. (2017). Reduction as an a posteriori relation. The British Journal for the Philosophy of Science.

Rueger, A. (2000). Physical emergence, diacronic and synchronic. Synthese, $124(3), 297-322$.

Schaffner, K. F. (1967). Approaches to reduction. Philosophy of Science, $34(2), 137-147$.

Schmelzer, J., \& Ulbricht, H. (1987). Thermodynamics of finite systems and the kinetics of first-order phase transitions. Journal of colloid and interface sceince, 117(2), 325-338.

Sornette, D. (2000). Critical phenomena in natural sciences. SpringerVerlag.

Wilson, K., \& Kogut, J. (1974). The renormalization group and the e expansion. Physics Reports, 12(2), 75-199.

Yin, M. (2011). Renormalization group transformations near the critical point: Some rigorous results. Journal of Mathematical Physics, 52(11), 113507. 